Appl. Set-Valued Anal. Optim. 4 (2022), No. 1, pp. 73-82

Available online at http://asvao.biemdas.com

https://doi.org/10.23952/asvao.4.2022.1.05

\title{
A NEW ALGORITHM FOR SOLVING STRONGLY MONOTONE EQUILIBRIUM AND MULTIVALUED VARIATIONAL INEQUALITY PROBLEMS
}

\author{
LE DUNG MUU ${ }^{1,2, *}$, NGUYEN VAN QUY ${ }^{3}$ \\ ${ }^{1}$ TIMAS, Thang Long University, Hanoi, Vietnam \\ ${ }^{2}$ Institute of Mathematics, VAST, Hanoi, Vietnam \\ ${ }^{3}$ Academy of Finance, 8 Phan Huy Chu, Hoan Kiem, Hanoi, Vietnam
}

\begin{abstract}
We propose a projection algorithm for solving strongly monotone equilibrium problems. An error bound for the algorithm is derived, and the application to a strongly multivalued monotone variational inequality is discussed. Some computational results are reported to show the efficiency and behavior of the proposed algorithm.

Keywords. Equilibrium problem; Error bound; Multivalued variational inequality; Strongly monotone bifunction.
\end{abstract}

\section{INTRODUCTION}

Let $\mathrm{C}$ be a nonempty closed convex subset in $\mathbb{R}^{n}$, and let $f: C \times C \rightarrow \mathbb{R}$ be a bifunction. We consider the equilibrium problem

$$
\text { find } x^{*} \in C: f\left(x^{*}, x\right) \geq 0, \quad \forall x \in C \text {. }
$$

As usual, we suppose that $f(x, x)=0$ for every $x \in C$, and the function $f(x,$.$) is convex, and sub-$ differentiable on $C$. Although this problem has a simple formulation, it contains many important problems, such as variational inequality problems, complementarity problems, fixed point problems, Nash equilibrium problems, and many others as special cases; see, e.g., $[1,2,3,4,5]$ and the references therein.

In recent years, many solution methods have been developed for solving Problem (EP) when $f$ possesses a certain monotonicity property. Strongly monotone equilibrium problem, which is a important class of the equilibrium problem, arises as subproblems in the proximal and Tikhonov regularization methods for ill-posed monotone problems; see, e.g., [6, 7, 8, 9]. A basis iterative method for solving strongly monotone problems and paramonotone equilibrium problems is the projection method, where, at current iterate $x^{k}$, the next iterate $x^{k+1}$ is defined by taking $x^{k+1}:=P_{C}\left(x^{k}-\alpha_{k} g^{k}\right)$ where the search direction $g^{k}$ is a certain point in the diagonal subdifferential $\partial_{2} f\left(x^{k}, x^{k}\right)$ of $f$ at $x^{k}$, and the stepsize $\alpha_{k}>0$. In order to ensure the convergence of $\left\{x^{k}\right\}$, the search direction $g^{k}$, and the step size $\alpha_{k}$ must satisfy certain additional conditions. In the algorithms proposed in $[10,11], g^{k}$ is required to be the projection of $x^{k}$ onto $\partial_{2} f\left(x^{k}, x^{k}\right)$,

${ }^{*}$ Corresponding author.

E-mail addresses: 1dmuu@ @ath.ac.vn (L.D. Muu), quynv2002@yahoo.com (N.V. Quy).

Received August 30, 2021; Accepted October 10, 2021.

(C)2022 Applied Set-Valued Analysis and Optimization 
however, the step size $\alpha_{k}$ much satisfy the condition $\sum_{k=1}^{\infty} \alpha_{k}^{2}<+\infty$ in [12, 13]. The first condition causes the difficulty when implementing the algorithm, while the second one makes the algorithm slowly convergent. In order to overcome these disadvantages, another method, called the auxiliary problem one, has been developed for strongly monotone equilibrium problems recently. In the auxiliary problem method, the iterate $x^{k+1}$ is defined as the unique solution of the strongly convex program

$$
\min \left\{f\left(x^{k}, y\right)+\frac{1}{\alpha_{k}}\left\|y-x^{k}\right\|, y \in C\right\} .
$$

To ensure the convergence, the stepsize $\alpha_{k}$ should satisfy certain conditions depending on the Lipschitz-type constant and the strong monotonicity modulus of the bifunction $f$ (see [12]). Note that if $f(x, y):=\langle F(x), y-x\rangle$, then Problem $(\mathrm{CP})$ reduces to $P_{C}\left(x^{k}-\alpha_{k} F\left(x^{k}\right)\right)$.

In this paper, we propose a projection algorithm for a strongly monotone equilibrium problem, where, at each iteration $k$, the search direction $g^{k}$ can be any point in $\partial_{2} f\left(x^{k}, x^{k}\right)$, and the step size $\alpha_{k}$ does not require to satisfy the condition $\sum_{k=1}^{\infty} \alpha_{k}^{2}<+\infty$. Moreover, in the case that $f$ is Lipschitz-type on $C$, an error bound for the algorithm is derived which could be used to estimate the number of iterations for obtaining an approximate solution.

The paper is organized as follows. The next section is devoted to the presentation of the algorithm and its convergence analysis. In the third section, we apply the proposed algorithm to the problem of finding an equilibrium point of a Walras model. We end the paper with some computational results on the model.

\section{Algorithm and Its Convergence Analysis}

First, let us recall some known concepts for the monotonicity properties of the bifunction.

Definition 2.1. Let $C$ be a nonempty closed convex set in $\mathbb{R}^{n}$ and let $f: C \times C \rightarrow \mathbb{R}$ be a bifunction. $f$ is said to be

(i) monotone on $C$ if

$$
f(x, y)+f(y, x) \leq 0, \quad \forall x, y \in C .
$$

(ii) strongly monotone on $C$ with modulus $\mu>0$ if

$$
f(x, y)+f(y, x) \leq-\mu\|x-y\|^{2}, \quad \forall x, y \in C .
$$

(iii) Lipschtz-type with constant $L_{1}, L_{2}>0$ on $C$ if

$$
f(x, y)+f(y, z) \geq f(x, z)-L_{1}\|x-y\|^{2}-L_{2}\|y-z\|^{2}, \quad \forall x, y, z \in C .
$$

It is known that if $f$ is strongly monotone then (EP) admits a unique solution (see [10]).

As usual, let us denote by $\partial_{2} f(x, x)$ the diagonal subdifferential of $f$ at $x \in C$ as the subdifferential of the convex function $f(x,$.$) at x$, that is,

$$
\partial_{2} f(x, x):=\{g:\langle g, y-x\rangle \leq f(x, y)+f(x, x) \forall y \in C\} .
$$

Is is known [14] that $f$ is strongly monotone (resp. monotone) on $C$ if and only if its diagonal subdifferential (as a multivalued mapping) is strongly monotone (resp. monotone).

We need the following lemma to prove the convergence of our algorithm. 
Lemma 2.1. [15] Let $\left\{\lambda_{k}\right\}$ be a sequence of positive numbers satisfying

$$
\lim _{k \rightarrow \infty} \lambda_{k}=0, \sum_{k=0}^{\infty} \lambda_{k}=+\infty,
$$

and let $\left\{\sigma_{k}\right\}$ be a sequence of real numbers satisfying $\lim _{k \rightarrow \infty} \sigma_{k}=0$. Assume that $\left\{a_{k}\right\}$ is a sequence of non-negative numbers such that

$$
a_{k+1} \leq\left(1-\lambda_{k}\right) a_{k}+\lambda_{k} \sigma_{k}, \quad \forall k \geq 1
$$

Then $\left\{a_{k}\right\}$ converges to 0 .

Ou algorithm is described as follows:

Algorithm 2.1. Choose a positive number sequence $\left\{\alpha_{k}\right\}$ such that

$$
\lim _{k \rightarrow \infty} \alpha_{k}=0 ; \sum_{k=0}^{\infty} \alpha_{k}=+\infty .
$$

Initial iteration. Choose $x^{0} \in C$ and set $k=0$.

Iteration $k=0,1, \ldots$

Step 1: Compute $g^{k} \in \partial_{2} f\left(x^{k}, x^{k}\right)$.

Step 2: Compute

$$
x^{k+1}:=P_{C}\left(x^{k}-\alpha_{k} g^{k}\right) .
$$

If $g^{k}=0$, then terminate: $x^{k}$ is the solution of the problem. Otherwise, replace $k$ by $k+1$ and go to Step 1 .

Proposition 2.1. Let $x^{*}$ be the unique solution of Problem (EP). Then, for every $k=0,1, \ldots$, the following inequalities hold true

$$
\left\|x^{k+1}-x^{k}\right\| \leq \alpha_{k}\left\|g^{k}\right\|
$$

and

$$
\left\|x^{k+1}-x^{*}\right\|^{2} \leq\left\|x^{k}-x^{*}\right\|^{2}+2 \alpha_{k} f\left(x^{k}, x^{*}\right)+2 \alpha_{k}^{2}\left\|g^{k}\right\|^{2} .
$$

Proof. From the property of the projection, it follows that

$$
\left\langle x^{k+1}-x^{k}+\alpha_{k} g^{k}, x^{k}-x^{k+1}\right\rangle \geq 0,
$$

which is equivalent to

$$
\left\|x^{k+1}-x^{k}\right\|^{2} \leq \alpha_{k}\left\langle g^{k}, x^{k}-x^{k+1}\right\rangle .
$$

Using the Cauchy-Schvarz inequality, we obtain (2.1).

By a simple algebraic manipulation, we have

$$
\begin{aligned}
\left\|x^{k+1}-x^{*}\right\|^{2}= & \left\|x^{k+1}-x^{*}-\left(x^{k+1}-x^{k}\right)\right\|^{2}-\left\|x^{k+1}-x^{k}\right\|^{2} \\
& +2\left\langle x^{k+1}-x^{*}, x^{k+1}-x^{k}\right\rangle \\
= & \left\|x^{k}-x^{*}\right\|^{2}-\left\|x^{k+1}-x^{k}\right\|^{2} \\
& +2\left\langle x^{k+1}-x^{*}, x^{k+1}-x^{k}\right\rangle \\
\leq & \left\|x^{k}-x^{*}\right\|^{2}+2\left\langle x^{k+1}-x^{*}, x^{k+1}-x^{k}\right\rangle .
\end{aligned}
$$

Again from the properties of the projection, one has

$$
\left\langle x^{k+1}-x^{k}+\alpha_{k} g^{k}, x^{*}-x^{k+1}\right\rangle \geq 0 .
$$


This inequality is equivalent to

$$
\begin{aligned}
\left\langle x^{k+1}-x^{*}, x^{k+1}-x^{k}\right\rangle & \leq \alpha_{k}\left\langle g^{k}, x^{*}-x^{k+1}\right\rangle \\
& =\alpha_{k}\left\langle g^{k}, x^{*}-x^{k}\right\rangle+\alpha_{k}\left\langle g^{k}, x^{k}-x^{k+1}\right\rangle .
\end{aligned}
$$

Using the convexity of $f\left(x^{k},.\right)$ and recalling $f\left(x^{k}, x^{k}\right)=0$, one has

$$
\left.\left\langle g^{k}, x^{*}-x^{k}\right\rangle \leq f\left(x^{k}, x^{*}\right)\right\rangle \text {. }
$$

Combining (2.1), and (2.3)-(2.5) we obtain

$$
\left\|x^{k+1}-x^{*}\right\|^{2} \leq\left\|x^{k}-x^{*}\right\|^{2}+2 \alpha_{k} f\left(x^{k}, x^{*}\right)+2 \alpha_{k}^{2}\left\|g^{k}\right\|^{2},
$$

which proves (2.2).

Theorem 2.1. Let $f$ be strongly monotone with modulus $\mu>0$, and let the diagonal subdifferential $\partial_{2} f(x, x)$ be bounded on $C$ for every $x \in C$. Then

(i) $\left\{x^{k}\right\}$ converges to $x^{*}$;

(ii) if, in additional, that $f$ satisfies the Lipschitz-type condition with constants $L_{1}, L_{2}>0$, where $L_{2}<\mu$, and that the diagonal subdifferential mapping $\partial_{2} f$ is Lipschitz-continuous with constant $L>0$ on a ball $B_{r}\left(x^{*}\right)$ with some $r>0$, then there exists an index $k_{0}$ (depending on $r$ ) such that

$$
\left\|x^{k+1}-x^{*}\right\|^{2} \leq \prod_{i=k_{0}}^{k}\left(\frac{1}{1+2 \sigma \alpha_{i}}\right)\left\|x^{k_{0}}-x^{*}\right\|^{2}, \forall k>k_{0}
$$

where $\sigma=\mu-L_{2}>0$.

Proof. (i) From (2.2), and the strongly monotonicity of $f$, we have

$$
\begin{aligned}
\left\|x^{k+1}-x^{*}\right\|^{2} \leq & \left\|x^{k}-x^{*}\right\|^{2}+2 \alpha_{k} f\left(x^{k}, x^{*}\right)+2 \alpha_{k} f\left(x^{*}, x^{k}\right) \\
& +2 \alpha_{k}^{2}\left\|g^{k}\right\|^{2} \\
\leq & \left\|x^{k}-x^{*}\right\|^{2}-2 \alpha_{k} \mu\left\|x^{k}-x^{*}\right\|^{2}+2 \alpha_{k}^{2}\left\|g^{k}\right\|^{2} .
\end{aligned}
$$

On the other hand, by the boundedness of the diagonal subdifferential $\partial_{2} f\left(x^{k}, x^{k}\right)$ for all $k$, it follows that there exists $\rho>0$ such that

$$
0<\left\|g^{k}\right\| \leq \rho, \forall k
$$

Combining (2.6), and (2.7), we obtain

$$
\begin{aligned}
\left\|x^{k+1}-x^{*}\right\|^{2} & \leq\left\|x^{k}-x^{*}\right\|^{2}-2 \alpha_{k} \mu\left\|x^{k}-x^{*}\right\|^{2}+2 \alpha_{k}^{2} \rho^{2} \\
& \leq\left(1-2 \mu \alpha_{k}\right)\left\|x^{k}-x^{*}\right\|^{2}+2 \alpha_{k} \mu\left(\frac{\rho^{2}}{\mu} \alpha_{k}\right) .
\end{aligned}
$$

From Lemma 2.1, we can deduce that $\lim _{k \rightarrow \infty} x^{k}=x^{*}$.

(ii) Let $r>0$. Since $x^{k} \rightarrow x^{*}$, we find that there exists $k_{0}$ such that $x^{k} \in B_{r}\left(x^{*}\right)$ for every $k>k_{0}$. For simplicity of notations, without loss of generality, we may assume that $k_{0}=0$. By applying the Lipschitz-type condition of $f$, we can write

$$
\begin{aligned}
f\left(x^{k}, x^{k+1}\right)+f\left(x^{k+1}, x^{*}\right) \geq & f\left(x^{k}, x^{*}\right)-L_{1}\left\|x^{k+1}-x^{k}\right\|^{2} \\
& -L_{2}\left\|x^{k+1}-x^{*}\right\|^{2}
\end{aligned}
$$


which is equivalent to

$$
\begin{aligned}
f\left(x^{k}, x^{*}\right)-f\left(x^{k}, x^{k+1}\right) \leq & f\left(x^{k+1}, x^{*}\right)+L_{1}\left\|x^{k+1}-x^{k}\right\|^{2} \\
& +L_{2}\left\|x^{k+1}-x^{*}\right\|^{2} \\
\leq & f\left(x^{k+1}, x^{*}\right)+f\left(x^{*}, x^{k+1}\right) \\
& +L_{1}\left\|x^{k+1}-x^{k}\right\|^{2}+L_{2}\left\|x^{k+1}-x^{*}\right\|^{2} \\
\leq & -\mu\left\|x^{k+1}-x^{*}\right\|^{2}+L_{1}\left\|x^{k+1}-x^{k}\right\|^{2} \\
& +L_{2}\left\|x^{k+1}-x^{*}\right\|^{2} .
\end{aligned}
$$

Since $g^{k+1} \in \partial_{2} f\left(x^{k+1}, x^{k+1}\right)$, we find by monotonicity of $f$ that

$$
\left\langle g^{k+1}, x^{k}-x^{k+1}\right\rangle+f\left(x^{k}, x^{k+1}\right) \leq 0 .
$$

Using (2.3), (2.4), (2.9), (2.10), the Lipschitz-type of $f$, and the Lipschitz property of the multivalued mapping $\partial_{2} f(.,$.$) , we can write$

$$
\begin{aligned}
\left\|x^{k+1}-x^{*}\right\|^{2} \leq & \left\|x^{k}-x^{*}\right\|^{2}+2 \alpha_{k} f\left(x^{k}, x^{*}\right)+2 \alpha_{k}\left\langle g^{k}, x^{k}-x^{k+1}\right\rangle \\
& -\left\|x^{k+1}-x^{k}\right\|^{2} \\
\leq & \left\|x^{k}-x^{*}\right\|^{2}+2 \alpha_{k}\left[f\left(x^{k}, x^{*}\right)-f\left(x^{k}, x^{k+1}\right)\right] \\
& +2 \alpha_{k} f\left(x^{k}, x^{k+1}\right)+2 \alpha_{k}\left\langle g^{k+1}, x^{k}-x^{k+1}\right\rangle \\
& +2 \alpha_{k}\left\langle g^{k}-g^{k+1}, x^{k}-x^{k+1}\right\rangle-\left\|x^{k+1}-x^{k}\right\|^{2} \\
\leq & \left\|x^{k}-x^{*}\right\|^{2}-2 \alpha_{k}\left(\mu-L_{2}\right)\left\|x^{k+1}-x^{*}\right\|^{2} \\
& -\left[1-2 \alpha_{k}\left(L+L_{1}\right)\right]\left\|x^{k+1}-x^{k}\right\|^{2} \\
\leq & \left\|x^{k}-x^{*}\right\|^{2}-2 \alpha_{k} \sigma\left\|x^{k+1}-x^{*}\right\|^{2},
\end{aligned}
$$

from which it follows that

$$
\begin{aligned}
\left\|x^{k+1}-x^{*}\right\|^{2} & \leq\left(\frac{1}{1+2 \sigma \alpha_{k}}\right)\left\|x^{k}-x^{*}\right\|^{2} \\
& \leq \prod_{i=0}^{k}\left(\frac{1}{1+2 \sigma \alpha_{i}}\right)\left\|x^{0}-x^{*}\right\|^{2} .
\end{aligned}
$$

Note that, under the assumption $\sum_{k=0}^{\infty} \alpha_{k}=+\infty$, it is easy to show that

$$
\prod_{k=0}^{\infty}\left(\frac{1}{1+2 \sigma \alpha_{k}}\right)=0,
$$

from which we can deduce that, for every $\varepsilon>0$, there exists an index $k_{\varepsilon} \in \mathbb{N}$ such that

$$
\prod_{i=0}^{k}\left(\frac{1}{1+2 \sigma \alpha_{i}}\right)\left\|x^{0}-x^{*}\right\|^{2}<\varepsilon \forall k>k_{\varepsilon} .
$$

Remark 2.1. From the strongly monotonicity of $f$ on $C$, it is not hard to see that if $u \in C$ and $d_{u} \in \partial_{2} f(u, u)$, then $\left\|x^{*}-u\right\| \leq \frac{\left\|d_{u}\right\|}{\mu}$, where $\mu$ is the strong monotonicity modulus of $f$ over $C$. Thus, in the algorithm, we can replace $C$ with $C \cap B_{R}(u)$ with $B_{R}(u)$ being the closed ball centered at $u$ with radius $R=\frac{\left\|d_{u}\right\|}{\eta}$. Then $\left\{x^{k}\right\}$ is bounded, and therefore, the conditions on theboundedness of $\left\{g^{k}\right\}$ and the Lipschiz continuity of the diagonal mapping can be omitted.

Next, we give an application to a multivalued variational inequality.

Let $F: \mathbb{R}^{n} \rightarrow \mathbb{R}^{n}$ be a multivalued mapping such that $\operatorname{dom} F \subseteq C$. The multivalued variational inequality problem is given as

$$
\text { find } x^{*} \in C, g^{*} \in F\left(x^{*}\right):\left\langle g^{*}, y-x^{*}\right\rangle \geq 0, \quad \forall y \in C \text {. }
$$


In the literature, there are many algorithms on solving single valued variational inequality problems; see, e.g., [6, 8, 16, 17, 18, 19, 20, 21, 22] and the references therein. We also mention here that there are rather few algorithms developed for multivalued variational inequality problems [9, 10, 24, 25, 26].

Suppose that $F(x)$ is compact and convex for every $x \in C$. Let

$$
f(x, y):=\max _{u \in F(x)}\langle u, y-x\rangle .
$$

Then $\partial_{2} f(x, x)=F(x)$. Moreover, $f$ is $\mu$-strongly monotone on $C$ if and only if $F$ is. In this case, Algorithm 2.1 reduces to the following one.

Algorithm 2.2. Choose a positive number sequence $\left\{\alpha_{k}\right\}$ such that

$$
\lim _{k \rightarrow \infty} \alpha_{k}=0, \sum_{k=0}^{\infty} \alpha_{k}=+\infty .
$$

Initial iteration. Choose $x^{0} \in C$ and set $k=0$.

Iteration $k=0,1, \ldots$.

Step 1: Compute $g^{k} \in F\left(x^{k}\right)$.

Step 2: Compute

$$
x^{k+1}:=P_{C}\left(x^{k}-\alpha_{k} g^{k}\right) .
$$

If $g^{k}=0$, then terminate: $x^{k}$ is the solution of the problem. Otherwise, replace $k$ by $k+1$ and go to Step 1.

Convergence results as well as the error bound for algorithm remain true for this algorithm.

\section{Some Numerical Results on a Walras Equilibrium Model}

Now we apply the proposed algorithms to solve Walras price equilibrium model originated by Walras [23]. The model is based on the equilibrium relations between supply and demand depending on prices. More precisely, the economy with perfect competition deals in $n$-commodities, with two stake holders, producers, and consumers. Given a price vector $p \in \Omega$, the producers (sectors) determine their supply $S(p) \subset \Omega$, while the consumers determine their demand $D(p) \subset \Omega$. Then the excess demand mapping is defined by

$$
E(p)=D(p)-S(p) .
$$

In some studies on price equilibrium models, the supply and/or demand mappings were given as the solution-set of certain optimization problems.

In the sequel, we are interest in the case (often in practice) when the price-set $\Omega$ of the commodity is given as

$$
\Omega:=\left\{p: p^{T}:=\left(p_{1}, \ldots, p_{n}\right): p_{i} \geq l_{p_{i}}>0, \forall i=1, \ldots, n .\right\}
$$

In this case, since $\Omega$ is not a cone, we formulate the model in the multivalued variational inequality problem

$$
\text { Find } p^{*} \in \Omega, q^{*} \in-E\left(p^{*}\right):\left\langle q^{*}, u-p^{*}\right\rangle \geq 0, \quad \forall u \in \Omega \text {. }
$$

There are various methods for solving this problem when the cost operator $-E$ is single valued and has certain monotonicity properties; see, e.g., $[6,8,14,15,17,18,19,20,21,22]$. When 
the cost operator is multivalued and monotone, the proximal regularization algorithms were commonly used $[9,10,24,25]$.

The problem of finding a Walras equilibrium price $p^{*}$ of this model then can be converted into the following multivalued variational inequality problem

$$
\text { find } p^{*} \in \Omega: \omega^{*} \in F\left(p^{*}\right),\left\langle\omega^{*}, u-p^{*}\right\rangle \geq 0, \quad \forall u \in \Omega \text {. }
$$

where $F(p)=S(p)-D(p)$ with $S$ (supply mapping) being the solution mapping of the problem of maximizing return of the producers that is given as

$$
\begin{aligned}
\max _{x} & p^{T} x \\
\text { subject to } & \left\{\begin{aligned}
A x & \leq b, \\
x & \geq 0, \quad p \in \Omega,
\end{aligned}\right.
\end{aligned}
$$

and $D$ (demand mapping) is the solution mapping of the consumer problem given by the solution-set of the mathematical program

$$
\begin{aligned}
\max _{x} & \left\{u(x)=A \prod_{i=1}^{n} x_{i}^{\alpha_{i}}\right\} \\
\text { subject to } & \left\{\begin{aligned}
p^{T} x & \leq M, \\
x & \in C_{d}, \quad p \in \Omega,
\end{aligned}\right.
\end{aligned}
$$

where $A=\left(a_{i j}\right)_{m \times n}$ is the technique matrix, $b \in \mathbb{R}^{m}$ is vector of input resources, $A, \alpha_{i}, i=1, \ldots, n$ are given positive numbers, and $M>0$ is the given budget level. Moreover, we suppose that $\Omega$ is the box in $\mathbb{R}^{n}$ given by

$$
\Omega:=\prod_{i=1}^{n}\left[l_{p_{i}}, u_{p_{i}}\right], l_{p_{i}}>0, \quad \forall i,
$$

and $C_{d}$ is the box in $\mathbb{R}^{n}$ given by

$$
C_{d}:=\prod_{i=1}^{n}\left[l_{x_{i}}, u_{x_{i}}\right], l_{x_{i}} \geq 0, \quad \forall i .
$$

Obviously, $u$ is a positively homogeneous with degree $\alpha=\sum_{i=1}^{n} \alpha_{i}>0$.

Set

$$
\begin{aligned}
\hat{\alpha} & :=\min \left\{\alpha_{i}, i=1, \ldots, n\right\}, \\
d^{2} & :=\max \left\{u_{x_{i}}^{2}: i=1, \ldots, n\right\} .
\end{aligned}
$$

We have the following lemma for the monotonicity of mappings $S$ and $D$.

Lemma 3.1. (i) The supply mapping $S$ is monotone (in general multivalued) on $\Omega$;

(ii) The mapping $-D$ is single-valued and inverse strongly monotone on $\Omega$ with modulus $\mu=\frac{\hat{\alpha} M}{\alpha d^{2}}$.

Proof. (i) For each $p \in \Omega$, denote by $S(p)$ the solution set of Problem (3.1). It is easy to see that $S(p)$ is nonempty for every $p \in \Omega$. Take two points $p^{1}, p^{2} \in \Omega$, and $x\left(p^{1}\right) \in S\left(p^{1}\right), x\left(p^{2}\right) \in$ $S\left(p^{2}\right)$. It follows that

$$
\begin{aligned}
& \left(p^{1}\right)^{T} x\left(p^{1}\right) \geq\left(p^{1}\right)^{T} x\left(p^{2}\right) \\
& \left(p^{2}\right)^{T} x\left(p^{2}\right) \geq\left(p^{2}\right)^{T} x\left(p^{1}\right) .
\end{aligned}
$$

Adding these two inequalities, we obtain

$$
\left\langle x\left(p^{1}\right)-x\left(p^{2}\right), p^{1}-p^{2}\right\rangle \geq 0, \quad \forall p^{1}, p^{2} \in \Omega .
$$

So, $S$ is monotone on $\Omega$. 
(ii) The problem defining $D$ is equivalent to the following strictly convex optimization problem

$$
\min _{x}\left\{p^{T} x-\frac{M}{\alpha} \sum_{i=1}^{n} \alpha_{i} \ln x_{i}: x \in C_{d}\right\} .
$$

Obviously, for every $p \in \Omega$, this problem has a unique optimal solution $x(p)$ with $x_{i}(p)>0$ for all $i=1, \ldots, n$. Take $p^{1}, p^{2} \in S$. From the optimality conditions for the convex program, we can write

$$
\begin{aligned}
& \left\langle p^{1}-\frac{M}{\alpha} \nabla\left(\sum_{i=1}^{n} \alpha_{i} \ln x_{i}\right)\left(x\left(p^{1}\right)\right), x\left(p^{1}\right)-x\left(p^{2}\right)\right\rangle \geq 0, \\
& \left\langle p^{2}-\frac{M}{\alpha} \nabla\left(\sum_{i=1}^{n} \alpha_{i} \ln x_{i}\right)\left(x\left(p^{2}\right)\right), x\left(p^{1}\right)-x\left(p^{2}\right)\right\rangle \geq 0 .
\end{aligned}
$$

Adding these two inequalities, we obtain

$$
\begin{aligned}
\left\langle-x\left(p^{1}\right)+x\left(p^{2}\right), p^{1}-p^{2}\right\rangle & \geq \frac{M}{\alpha} \sum_{i=1}^{n} \frac{\alpha_{i}\left[x_{i}\left(p^{1}\right)-x_{i}\left(p^{2}\right)\right]^{2}}{x_{i}\left(p^{1}\right) x_{i}\left(p^{2}\right)} \\
& \geq \frac{M \hat{\alpha}}{\alpha d^{2}}\left\|x\left(p^{1}\right)-x\left(p^{2}\right)\right\|^{2}, \forall p^{1}, p^{2} \in S .
\end{aligned}
$$

So, the mapping $-D$ with $-D(p)=-x(p)$ is strongly inverse monotone on $\Omega$.

It is easy to see that, for each $p \in \Omega$, the optimal solution of Problem (3.3) is given by

$$
\left\{\begin{array}{l}
x_{i}(p)=\frac{M \alpha_{i}}{\alpha p_{i}} \text { if } \frac{M \alpha_{i}}{\alpha p_{i}} \leq u_{x_{i}} \\
x_{i}(p)=u_{x_{i}} \text { if } \frac{M \alpha_{i}}{\alpha p_{i}}>u_{x_{i}}, i=1, \ldots, n .
\end{array}\right.
$$

In order to solve Problem (MVI), we apply the proximal algorithm presented in [9]. In this algorithm, at each iteration $k$, we have to solve the strongly monotone multivalued variational inequality problem over $\Omega$, where the cost mapping is

$$
\Phi\left(u, D_{\lambda}\left(p^{k}\right)\right)=S(u)+\frac{1}{\lambda_{k}}\left(u-D_{\lambda}\left(p^{k}\right)\right),
$$

with

$$
\lambda_{k}>0, D_{\lambda}\left(p^{k}\right)=p^{k}+\lambda_{k} D\left(p^{k}\right) .
$$

In the table below, we use the following headings

- $N$ : the number of the tested problems;

- $n$ : the number of types of goods;

- Average iter 1 : the average number of iterations for solving one strongly monotone inequality;

- Average time 1: the average time (in second) needed to solve one strongly monotone inequality;

- Average iter2: the average number of iterations for solving one problem to find an equilibrium price;

- Average time2: the average time (in second) needed to solve one problem to find an equilibrium price;

All other input data were generated randomly. 


\begin{tabular}{|c|c|c|c|c|c|}
\hline $\mathrm{N}$ & $\mathrm{n}$ & Average iter 1 & Average time 1 & Average iter 2 & Average time 2 \\
\hline 10 & 5 & 636 & 5.33 & 3 & 15.98 \\
10 & 10 & 973 & 6.71 & 18 & 120.73 \\
10 & 20 & 1949 & 15.35 & 6 & 92.13 \\
10 & 30 & 3050 & 6.25 & 4 & 99.25 \\
10 & 40 & 5974 & 62.46 & 3 & 187.38 \\
10 & 50 & 4677 & 50.94 & 3 & 152.81 \\
10 & 100 & 5117 & 79.13 & 2 & 158.25 \\
10 & 150 & 5249 & 82.03 & 3 & 246.08 \\
10 & 200 & 6001 & 116.66 & 4 & 466.63 \\
\hline
\end{tabular}

\section{REFERENCES}

[1] G. Bigi, M. Castellani, M. Pappalardo, M. Passacantando, Nonlinear Programming Techniques for Equilibria, Springer-Verlag, New York, 2019.

[2] S.Y. Cho, A monotone Bregan projection algorithm for fixed point and equilibrium problems in a reflexive Banach space, Filomat, 34 (2020), 1487-1497.

[3] Y. Zheng, J. Shi, A splitting method for a family of equilibrium and inclusion problems, J. Nonlinear Funct. Anal. 2020 (2020), Article ID 44.

[4] N. Shahzad, H. Zegeye, Convergence theorems of common solutions for fixed point, variational inequality and equilibrium problems, J. Nonlinear Var. Anal. 3 (2019), 189-203.

[5] L.C. Ceng, A subgradient-extragradient method for bilevel equilibrium problems with the constraints of variational inclusion systems and fixed point problems, Commun. Optim. Theory 2021 (2021), Article ID 4.

[6] F. Facchinei, J.S. Pang, Finite-Dimensional Variational Inequalities and Complementary Problems, SpringerVerlag, New York, 2003.

[7] P.G. Hung, L.D. Muu, The Tikhonov regularization extended to equilibrium problems involving pseudomonotone bifunctions, Nonlinear Anal. 74 (2011), 6121-6129.

[8] I. Konnov, Combined Relaxation Methods for Varaitional Inequalities, Springer, 2011.

[9] R.T. Rockafellar, Monotone operators and the proximal point algorithm, SIAM Control Optim. 14 (1976), 877-898.

[10] P.N.Anh, L.D. Muu, V.H. Nguyen, J. J. Strodiot, Using the Banach contraction principle to implement the proximal point method for multivalued monotone variational inequalities, J. Optim. Theory Appl. 124 (2005), 285-306.

[11] T.V. Thang, Inertial subgradient projection algorithm for solving pseudomonotone equilibrium problems and it's convergence rate, Bulletin Iranian Math. Soc. (2021), to appear.

[12] P.M. Duc, L.D. Muu, N.V. Quy, solution-existence and algorithms with their convergence rate for strongly pseudomonotone equilibrium problems, Pacific J. Optim. 12 (2016), 833-845.

[13] P. Santos, S. Scheiberg, An inexact subgradient algorithm for equilibrium problems, Comput. Appl. Math. 30 (2011), 91-107.

[14] T.D. Quoc, L.D. Muu, V.H. Nguyen, Extragradient algorithms extended to equilibrium problems, Optimization 57 (2008), 749-776.

[15] S. He, H.K. Xu, Variational inequalities governed by boundedly Lipschitzian and strongly monotone operators, Fixed Point Theory 10 (2009), 245-258.

[16] L.V. Nguyen, X. Qin, Some results on strongly pseudomonotone quasi-variational inequalities, Set-Valued Var. Anal. 28 (2020), 239-257.

[17] D.V. Hieu, P.K. Anh, L.D. Muu, Modified hybrid projection methods for finding common solutions to variational inequality problems, Comput. Optim. Appl. 66 (2017), 75-96.

[18] D.V. Hieu, D.V. Thong, New extragradient-like algorithms for strongly pseudomonotone variational inequalities, J. Glob. Optim. 70 (2018), 385-399.

[19] D. Hieu, J.J. Strodiot, L.D. Muu, An explicit extragradient algorithm for solving variational inequalities, J. Optim. Theory Appl. 185 (2019), 476-503. 
[20] P.D. Khanh, P.T. Vuong, Modified projection method for strongly pseudomonotone variational inequalities, J. Glob. Optim 58 (2014), 341-350.

[21] Y. Malitsky, Projected relected gradient methods for monotone variational inequalities, SIAM J. Optim. 25 (2015), 502-520.

[22] Y. Shehu, Q.-L. Dong, D. Jiang, Single projection method for pseudomonotone variational inequality in Hilbert spaces, Optimization 68 (2018), 385-409.

[23] L. Walras, Elements d'Economie Politique Pure, L. Corbaz, Lausanne; English translation: Elements of pure economics, Allen and Unwin, London, 1954.

[24] J.C. Yao, Multivalued variational inequalities with K-pseudomonotone operators, J. Optim. Theory Appl. 83 (1994), 391-403.

[25] Q.L. Dong, Y.Y. Lu, J. Yang, S. He, Approximately solving multivalued variational inequalities by using a projection and contraction algorithm, Numer. Algo. 76 (2017), 799-812.

[26] J. Yang, J. Lui, Strong convergence result for solving monotone variational inequalities in Hilbert space. Numer. Algo. 80 (2019), 741-752. 